\title{
Aplícate: potenciación del trabajo colectivo mediante la realización de prácticas utilizando las nuevas tecnologías ${ }^{1}$
}

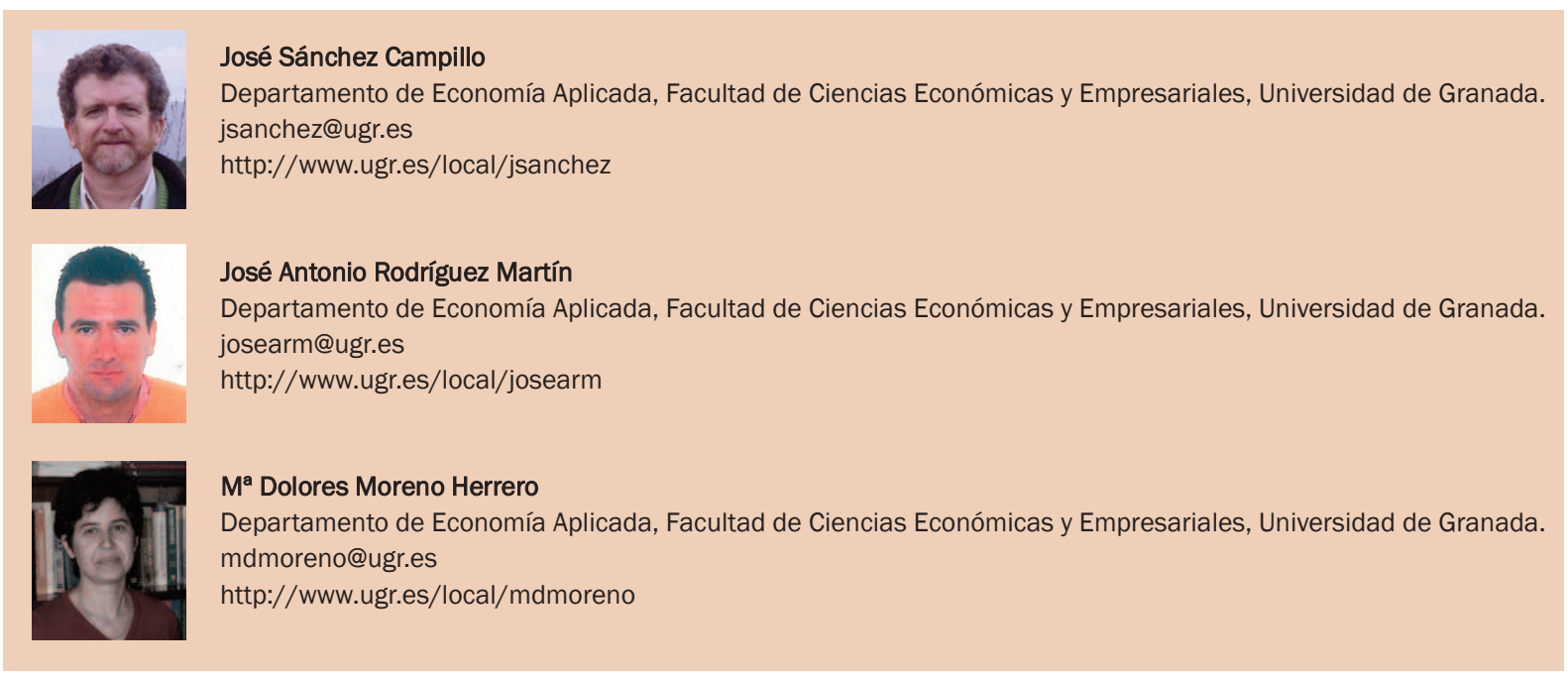

|Fecha presentación: 18/01/2011 | Aceptación: 05/04/2011 | Publicación: 21/06/2011

\begin{abstract}
1. Introducción y objetivos
La plataforma web Aplícate (http://www.aplicate.net) fue creada en el curso 2004/05, gracias a un Proyecto de Innovación docente (Potenciación del Trabajo Colectivo Mediante la Realización de Prácticas Utilizando las Nuevas Tecnologías), impulsado por el profesor José Sánchez Campillo, y aprobado y financiado por el Vicerrectorado de Planificación, Calidad y Evaluación Docente de la Universidad de Granada. Esta plataforma cuenta con el ISBN 84-689-4009-7, y con la autorización de la Agencia de Protección de Datos (contiene ficheros con los datos personales de los estudiantes, que son habituales en la tradicional ficha de clase).

Desde el año 2005, ocupa la primera posición en Google, al realizar una búsqueda con el término "aplicate". Este lugar tan privilegiado en el buscador más utilizado se ha podido alcanzar y mantener, hasta el momento, en virtud de su elevado nivel de utilización por parte de los estudiantes y profesores.

Además, el desarrollo de la web ha servido para incluir en la misma otros dos proyectos de innovación, coordinados por el profesor José Sánchez Campillo: El de innovación docente,
\end{abstract}

Trabajos Colectivos Utilizando las Nuevas Tecnologías de la Información y Comunicación, y el de Innovación en Tutorías, Empléate, que se vienen desarrollando desde el curso 2005/o6.

Entre los objetivos de la plataforma Aplícate, figuran el servir de punto de encuentro para compartir información y recursos (programas de asignaturas, documentos, protocolos de trabajos, datos de rendimiento académico, vídeos educativos, etc.), especialmente de cara a la implementación del Espacio Europeo de Educación Superior (EEES).

La plataforma también proporciona soporte informático a todos los profesores que quieran participar en la misma (actualmente veintisiete), siempre que exista el compromiso por parte del docente de poner a disposición de los alumnos un determinado nivel de información, en formato electrónico, para el desarrollo de las clases teóricas y prácticas. Hay que subrayar que la plataforma no sustituye en ningún caso a la web personal de cada profesor, ni al tablón de docencia de la Universidad de Granada, sino que es un instrumento totalmente complementario a los ya existentes.

En definitiva, en el nuevo contexto educativo, con la re-

${ }^{1}$ Este proyecto se puso en marcha en el curso 2004/05, gracias a un Proyecto de Innovación Docente financiado por el Vicerrectorado de Planificación, Calidad y Evaluación Docente de la Universidad de Granada, patrocinado, asimismo, por el Departamento de Economía Aplicada de la Universidad de Granada. 
forma de los planes de estudio, de acuerdo con los principios que inspiran el EEES, es conveniente impulsar una nueva orientación de las clases teóricas y prácticas. Con este proyecto, se pretende, entre otras actuaciones, desarrollar los protocolos para la realización de trabajos de los estudiantes. Esta iniciativa es claramente complementaria al Plan de Innovación en Tutorías Empléate comentado, porque los trabajos que realizan los estudiantes durante su periodo de estudio en la universidad deben contribuir positivamente a la mejora de su empleabilidad, una vez que consigan su graduación. En este sentido, casi todos los profesores que participan en Aplícate también son miembros activos del Plan Empléate.

\section{Método y análisis}

Hasta el momento, el menú principal de la web http://www.aplicate.net cuenta con siete grandes apartados (página principal, participantes, documentación, patrocinadores, acceso restringido, contacto y enlaces).

Los participantes en el Plan de Innovación disponen de diversos recursos que se han ido desarrollando desde la creación de la página web. Ésta constituye el soporte de los archivos de documentación docente en formato electrónico, que cada curso académico se van alojando y actualizando.

En concreto, los contenidos de tales archivos engloban: los programas de las asignaturas, la bibliografía, las lecturas y los ficheros de datos, los artículos, revistas y libros, los protocolos de actuación para los trabajos prácticos, los enunciados y soluciones de las prácticas aportadas por los estudiantes y comentadas por los profesores. Asimismo, se incorporan las grabaciones digitales de la exposición de los trabajos de los estudiantes, modelos de exámenes finales, las calificaciones concedidas por los estudiantes a sus compañeros y las finalmente concedidas por el profesorado, estadísticas de participación y de resultados académicos, sugerencias y recomendaciones de estudiantes, libretas de direcciones de correo electrónico de los estudiantes, fichas y otros materiales. Para salvaguardar el derecho a la intimidad, y cumplir con la Ley de Protección de Datos, muchos de estos recursos están en la plataforma con acceso restringido (estudiantes, profesores y administrador).

Hay que destacar un nuevo apartado en el que aparecen los protocolos de los trabajos que se piden a los estudiantes, así como la justificación de los mismos por su contribución a los conocimientos, capacidades y destrezas del correspondiente título que está cursando el estudiante (contenidos en las fichas técnicas de las propuestas de título de grado según RD 55/2005, de 21 de enero y/o los libros blancos correspondientes de la Agencia Nacional de Evaluación de la Calidad y Acreditación (ANECA). También se especifica el tiempo que, en promedio, deben dedicar los estudiantes para realizar los trabajos con éxito.

Para la mejor visualización y difusión de los resultados del proyecto, se ha creado un menú con los siguientes contenidos: a) los protocolos, que sirven para la realización de las clases prácticas y teóricas, debidamente clasificados por titulaciones; b) los trabajos más destacados de los estudiantes clasificados por asignaturas; y c) los vídeos de las exposiciones de los trabajos que se consideren de mayor interés.

Particularmente, el Plan de Innovación se fundamenta, de forma secuencial, en cada curso académico, en la siguiente metodología de trabajo:

1. Presentación por parte de los profesores de las asignaturas y de los protocolos para la realización de trabajos por parte de los estudiantes al resto de profesores participantes en el proyecto, suscitando una discusión sobre la adecuación de los mismos.

2. Creación de grupos de trabajo, en base a la afinidad de las asignaturas implicadas en el proyecto, para el diseño de los trabajos que se proponen a los estudiantes.

3. Redacción final de los nuevos protocolos por parte de los profesores, así como la justificación de los mismos, por su posible contribución a los conocimientos, capacidades y destrezas del correspondiente título que está cursando el estudiante. Estos protocolos serán leídos por los estudiantes que participan en el proyecto, con el fin de comprobar que su redacción es comprensible por los mismos y, en su caso, introducir los cambios que sean pertinentes.

4. Exposición de los trabajos de los estudiantes de las asignaturas y grabación en vídeo o cámara digital de la mayoría de los mismos, según criterio de cada profesor.

5. Evaluación de los trabajos efectuados por los estudiantes y selección de los más destacados por parte del profesorado.

6. Análisis y evaluación de las actividades realizadas y propuestas de mejora para los cursos siguientes.

7. Reestructuración de la web http://www.aplicate.net, en función de los nuevos ficheros disponibles.

En general, los profesores que imparten las asignaturas de los últimos cursos tienen más experiencia al respecto que los que imparten las de los primeros cursos. Por esta razón, es esencial que durante el primer cuatrimestre, y en primer lugar, los profesores que ya tienen protocolos bastante elaborados, sean los que presenten y justifiquen el contenido de los trabajos que se les exigen a los estudiantes, ya sea en las asignaturas del primer o segundo cuatrimestre.

A partir de la discusión de estos protocolos ya elaborados, y de las Guías ECTS de las asignaturas, se debe diseñar una plantilla general para la petición de trabajos a los estudiantes, que pueda servir de base para todas las asignaturas. Una vez elaborada dicha plantilla, se crean grupos de trabajo para que, con independencia del cuatrimestre en el que se imparta cada asignatura, al final del curso se tengan protocolos para los trabajos de todas las asignaturas implicadas en el proyecto.

En consecuencia, al final de cada curso existirán protocolos y trabajos realizados por los estudiantes para las asignaturas, así como las grabaciones en vídeo de sus exposiciones. A partir de este material, cada profesor seleccionará los trabajos más representativos y/o destacados de sus estudiantes, y los vídeos de mayor interés. La profesora Dolores Moreno Herrero es la encargada de que dicho material, debidamente clasificado y contando con la colaboración de los estudiantes y profesores, se aloje adecuadamente en la web.

\section{Resultados y Discusión}

El número de estudiantes participantes en la Plataforma Aplícate (http://www.aplicate.net), se ha incrementado, año tras año, debido a que cada vez hay más profesores y asignaturas dentro del proyecto. En el pasado curso se inscribieron 1.400 estudiantes, de tal modo que, desde el inicio de la Plataforma, se han registrado más de 6.000 estudiantes.

La inscripción se realiza fácilmente desde el acceso restringido mediante una misma ficha electrónica, que es común para todos los profesores que están participando en el proyecto. En la misma, el estudiante debe insertar el correo electrónico que habitualmente utiliza, y no necesariamente el de la universidad, por lo que recibirá los correos electrónicos de 
los profesores participantes. Asimismo, el estudiante introduce normalmente en su ficha un número de móvil, con lo que por medio de un SMS o una llamada, el profesor puede contactar con sus estudiantes con mayor rapidez.

En la plataforma se dispone de documentación, tanto en abierto como mediante el acceso identificado, de más de 30 asignaturas, de diferentes titulaciones y facultades de la Universidad de Granada. Como indicador de la utilidad de la misma debe mencionarse que, después de conseguir la graduación, muchos ex alumnos continúan dados de alta en la Plataforma Aplícate. Se trata de una forma sencilla y ágil que permite poder recibir ofertas de empleo y estar puntualmente informados de las sesiones informativas sobre inserción laboral y creación de empresas, que se realizan frecuentemente en la Facultad de Ciencias Económicas y Empresariales y otros centros de la Universidad de Granada.

Así, los beneficios del proyecto no recaen en los estudiantes y profesores de una única titulación, sino que son beneficiarios los estudiantes y profesores de titulaciones que se imparten en la Facultad de Ciencias Económicas y Empresariales, de Ciencias, de Derecho, de Ciencias del Trabajo y de Ciencias Políticas.

De este modo, el carácter transversal del proyecto, en cuanto a número de titulaciones y tipo de estudiantes que se ven afectados por el mismo, se deriva de que participan en el Plan de Innovación profesores con docencia en todas las titulaciones relacionadas.

Finalmente, en función de sus destinatarios, cabe señalar los siguientes resultados del proyecto:

1) Para los estudiantes: a) Incremento del nivel de conocimientos sobre las nuevas tecnologías de la información y comunicación; b) aumento de la capacidad para conseguir información y resolver los problemas en fechas concretas; c) incremento de la capacidad de comunicación, de síntesis y de exponer ideas en tiempo limitado; d) potenciación de la iniciativa y la responsabilidad individual; e) desarrollo de la capacidad de trabajar en grupo; f) aumento de la experiencia para afrontar situaciones de presión externa y hablar con naturalidad en un auditorio concurrido.

2) Para los profesores: a) se favorece el intercambio de información, materiales y experiencias educativas con otros profesores, que se enfrentan a entornos educativos similares; b) se minimizan los efectos del habitual individualismo existente en la labor docente, que provoca la disipación de los recursos y la descoordinación entre las diversas asignaturas; c) mayor coordinación en el proceso de elaboración y difusión de la información, apuntes, transparencias, vídeos, y otros materiales educativos, por parte de los profesores implicados en este proyecto de innovación.

3) Para los estudiantes y profesores conjuntamente: a) incremento de la comunicación e interacción entre los estudiantes y los profesores, y, a su vez, entre los miembros de cada uno de esos colectivos; b) se favorece la actualización de los contenidos de las asignaturas y de los trabajos de los estudiantes, de acuerdo con la evolución de las nuevas tecnologías; c) incorporación de las sugerencias de los estudiantes y los cambios que se producen en los programas de otras asignaturas; d) se incrementa el peso del contenido práctico de las asignaturas que participan en el Plan de Innovación.
Desde una óptica temporal, la mayoría de los profesores que suscriben este proyecto llevan utilizando la plataforma Aplícate desde hace varios años, de manera que la vigencia del proyecto sobrepasa el de un curso académico por diversos motivos, a saber:

En primer lugar, porque los objetivos y productos que se pretenden alcanzar con el mismo se mantendrán en vigor, curso tras curso, incluso se ven reforzados en el contexto del EEES.

En segundo lugar, su continuidad y proyección se ve favorecida, porque determinados recursos ya generados con el proyecto de innovación perduran varios cursos, prácticamente sin costes adicionales de mantenimiento en su alojamiento, mejora y actualización; así, por ejemplo, las fichas de los alumnos que cada curso participan en el Plan de Innovación, se pueden mantener activas durante los cursos correspondientes hasta completar la titulación, con el único requisito para el estudiante de marcar en cada curso las asignaturas en que se encuentra matriculado, y actualizando, si fuera necesario, cualquier otro dato personal que hubiese cambiado.

En tercer lugar, la prolongación del plan permite generar una base de datos con la dirección de correo electrónico y de número de móvil de los estudiantes, que, posteriormente, facilitará el seguimiento de los estudiantes y graduados en su proceso de inserción laboral. En esta línea, el cuestionario on-line, vinculado a la página web del propio proyecto y de la Facultad de Empresariales, ha permitido realizar el Estudio de la Inserción Laboral de sus Graduados en el curso 2005-2006.

\section{Evaluación del proyecto y conclusiones}

El propósito del proyecto es que los beneficios del mismo no recaigan exclusivamente en los estudiantes y profesores de una única titulación y/o facultad, sino que se extiendan a los estudiantes y profesores de otras titulaciones y centros de la Universidad de Granada.

La continuidad de Aplícate se ve impulsada por el propio interés de los profesores, en cuanto que les permite rentabilizar el esfuerzo que cada curso han venido realizando, con el fin de incrementar y mejorar las aportaciones a la plataforma, ya sea de materiales, ejercicios u otros recursos, en el marco del EEES.

La plataforma impulsa, desde su creación, un sistema de comunicación permanente, en el que se integran como recursos las diversas posibilidades educativas, no sólo del correo electrónico, sino también del tablón de docencia de la Universidad de Granada y de las páginas web de los profesores participantes en el Plan de Innovación Aplícate.

Se cuenta con varios recursos de evaluación incluidos en la propia página web, en cuyo menú principal se dispone de la opción de contacto, por medio de la cual cualquier participante en el Plan de Innovación puede realizar los comentarios, sugerencias, propuestas o valoraciones que considere oportunos respecto a su desarrollo y a la contribución de éste a los objetivos que pretende conseguir. Asimismo, en dicho menú también se dispone de la opción Blog, que abre la posibilidad de, a través de la propia plataforma, aportar las opiniones y valoraciones que contribuyan a mejorar las potencialidades de Aplícate.

A la vista de los resultados del citado Estudio de la Inserción Laboral de los Graduados de la Universidad de Granada, este plan ha sido muy bien valorado por los estudiantes. La mejora que pretende el proyecto se plasma en el desarrollo de determinadas competencias, aptitudes y acti- 
tudes, que revierten positivamente en el rendimiento académico del alumno, y que hace más rentable su inversión en educación superior, facilitando su integración en el mercado de trabajo. En este sentido, cabe señalar que existe una opinión generalizada entre los estudiantes sobre la necesidad de desarrollar actividades orientadas a la inserción laboral.

Concluyendo, con este proyecto se ha impulsado la utilización de las nuevas tecnologías de la información y comunicación, que demanda la sociedad del conocimiento, y pretendemos desarrollar las competencias contempladas en el Libro Blanco de los diferentes Grados. En este contexto, en cursos anteriores, se ha potenciado con Aplícate el uso común de la información y el desarrollo de recursos didácticos, de acuerdo con los principios y métodos docentes sobre los que se asientan los pilares del EEES, cuya adaptación constituye el gran reto de la universidad para la primera mitad del siglo XXI.

Además, se han mejorado, entre otros aspectos, la flexibilidad y adaptabilidad de los estudiantes ante nuevas situaciones que requieren nuevos métodos de trabajo, así como la capacidad de los profesores para trabajar en equipo y, en esencia, se ha intensificado la preocupación por la calidad docente por parte de los miembros de este proyecto.

\section{Bibliografía}

ANECA (2005). Libro blanco. Título de grado en Economía y Empresa. Madrid: Agencia Nacional de Evaluación de la Calidad y Acreditación.

Bain, Ken (2006). Lo que hacen los mejores profesores universitarios. Valencia: Publicacions de la Universitat de València.

Balsamo, Anne (2010). Our approach to pedagogy must shift from being teacher-centered to being learner-centered, @tic. Revista d'innovació educativa.4, pp. 86-88. http://ojs.uv.es/index.php/attic/article/viewPDFInterstitial/180/197

Clark, Andrew (2001). What really matters in a job? Hedonic measurement using quit data, Labour Economics, 8, pp. 223-242.

Gamero Carlos. (2006). Efectos de la satisfacción laboral y de la educación sobre la búsqueda de empleo desde el empleo. En: Moreno, Dolores y Sánchez, José. (Coord.): Investigaciones de Economía de la Educación, 1. Granada, Asociación de Economía de la Educación y Copicentro, pp. 337-353.

Moreno, Dolores et al. (2008). Estudio de la inserción laboral de los graduados en el curso 2005-06 en la Facultad de Ciencias Económicas y Empresariales de la Granada: Universidad de Granada.

Página Web del Proyecto de Innovación Docente Aplicate: http://www.aplicate.net
Rodríguez, José Antonio et al. (2009). Aplicate: Potenciación del trabajo colectivo mediante la realización de prácticas utilizando las nuevas tecnologías, 1as. Jornadas de innovación docente universitaria, Córdoba: Agencia Andaluza de Evaluación (AGAE).

Sánchez, José (2009). Plan de Innovación Docente Trabajos Colectivos Utilizando las Nuevas Tecnologías de la Información y Comunicación. 2005-2008, www.aplicate.net, Granada: Universidad de Granada.

Villa, M. (2004). Educadores orientados al aprendizaje. En: F. Michavila y J. Martínez (Eds). La profesión de profesor de universidad. Madrid: Cátedra UNESCO de Gestión Política, 2004, pp. 53-60. 